\title{
Assessment of Tomato Advanced Lines to Resistance of Late Blight
}

\author{
P.K. Ray $^{1 *}$, R.B. Verma ${ }^{2}$, S.S. Solankey ${ }^{2}$ and A. Chaudhary ${ }^{3}$ \\ ${ }^{1}$ Subject Matter Specialist (Horticulture), K. V. K., Saharsa, Bihar, India \\ ${ }^{2}$ Department of Horticulture (Veg. \& Flori.), B.A.U., Sabour, Bhagalpur, Bihar, India \\ ${ }^{3}$ Subject Matter Specialist (Plant Breeding \& Genetics), K. V. K., Saharsa, Bihar, India \\ *Corresponding author
}

\begin{abstract}
A B S T R A C T
Keywords

Phytophthora infestans, Tomato,

resistance and late blight disease

Article Info

Accepted:

20 December 2017

Available Online:

10 January 2018

Experiment was conducted to screen different advanced lines and genotypes in line $\times$ tester mating design against late blight of tomato caused by Phytophthora infestans. One hundred genotypes were screened during winter season under field condition and data was recorded on 0 - 5 scale at 30, 60 and 90 days in which eleven genotypes were highly resistant, seventeen genotypes were resistant, nineteen genotypes were moderately resistant, twenty four genotypes were susceptible and twenty nine genotypes were highly susceptible. Forty eight $\mathrm{F}_{1} \mathrm{~s}$ were screened along with their parents during rainy season, 2014 under field condition. Out of forty eight $F_{1}$ s, eighteen cross combinations were highly resistant and others were resistant, moderately resistant, susceptible and highly susceptible. The parents namely Solanum peruvianum and Pusa Rohini showed highly resistant and other parents were either resistant, moderately resistant, susceptible or highly susceptible in respect of disease reaction.
\end{abstract}

\section{Introduction}

Tomato (Lycopersicon esculentum Mill) is an important vegetable of exceptionally high nutritive value and versatile food use (Afroz et al., 2009; Saleem et al., 2009; Noureen et al., 2010). Late blight, caused by the oomycete pathogen Phytophthora infestans (Mont.) de Bary, is an economically important disease of tomato (L. esculentum) worldwide (Majid et al., 1992; Yan et al., 2002). The causal pathogen from tomato was first described by Payen in France in 1847 (Payen, 1847) and has been found responsible for numerous epidemics since it was first described
(Stevenson, 1997). P. infestans has a wider host range which includes $L$. esculentum, $S$. tuberosum, S. sarrachoides, S. triflorum, $S$. dulcamara, S. sisymbriifolium, Nicotiana benthamiana and plants of the genus Calibrachoa (Bectell et al., 2006; Dandurand et al., 2006; Flier et al., 2003; Lebecka, 2008). $P$. infestans can attack leaves, petioles, stems, fruits and seeds of tomato (Irzhansky and Cohen 2006). Late blight disease may be initiated in nursery and adult plants by airborne sporangia or by oospores harboring the soil and seed (Rubin and Cohen, 2004; Govers, 2005). Disease symptoms may start as water soaked, pale green irregular leaf lesions, which enlarge, turn brown, shrivel and dry 
out. Under conditions of moist weather, the underside of the lesions may be covered with a fine white moldy growth composed of sporangiophores and sporangia. On petioles and stems lesions appear at any point as oily, brown areas later turning into black and the whole plant may die. On fruits the disease appears as dark green to brown, greasy, irregular blotches, and fruit become shriveled at later stages. Cool, rainy weather, high relative humidity and heavy dew formation favor the infection, disease progress and sporangia production (Mohan et al., 1996; Stevenson, 1997) which can destroy the unprotected crop within 10 to 14 days (Rubin and Cohen, 2004; Govers, 2005). Diseasemanagement strategies mainly depend on fungicide applications, which are uneconomical and less effective due to increasing resistance of the pathogen against fungicides (Griffith et al., 1992). Identification and utilization of genetic resources resistant to $P$. infestans in tomato is the only way to develop late bight-resistant tomato cultivars following appropriate breeding methods. Although vast genetic diversity exists in well adapted cultivars/germplasm in tomato in India, so far no systematic study on resistance or susceptibility level of existing tomato genetic resources has been conducted. The main objective of the present investigation was to determine the level of resistance in cultivated and wild Solanaceous species to identify potential germplasm resistant to late blight disease. Such information would help breeders to develop blight resistant cultivars.

\section{Materials and Methods}

In order to find out the resistant source against the late blight of tomato were evaluated under natural conditions. The experiment was conducted at Vegetable Research Farm, Bihar Agricultural University, Sabour, Bhagalpur, Bihar, India. Total one hundred tomato genotypes representing cultivars and wild accessions of Solanum species were screened under field condition during winter seasons 2012. After screening of one hundred genotypes selected twelve lines and four testers under line $\times$ tester mating design. The experimental material comprising of forty eight $F_{1}$ hybrids and sixteen parental lines were transplanted in the field in a Randomized Block Design with three replications in next year. In each replication, there were ten plants in a row for each entry. The parents were grown during spring seasons, 2013 in crossing block. After screening the twelve genotypes of tomato namely Arka Vikas, H-86 (Kashi Vishesh), Arka Meghali, LA-3967 (IIHR2374), LA-3976 (IIHR-2381), LA-3938 (IIHR-2347), LA-3962 (IIHR-2370), H-88-785, LA-3952 (IIHR-2361), LA-3948 (IIHR2357), LA-3930 (IIHR-2339), Pant-T-5 selected as female parents and four genotypes viz., IIHR-2195 (IC-395457), Solanum peruvianum, IIHR-2199 (IC-395461), Pusa Rohini were used as male parents for the cross.

The natural screenings were performed during the period when the conditions were favorable for white fly perpetuation that had natural pressure on entire germplasm.

The screening was done at 30, 60 and 90 days after transplanting during each year of experimentation under field conditions after appearance of symptom. The symptom severity was recorded at a 0-5- scale on each genotype following the method described by (Akhtar et al., 2012) with certain modifications.

Disease incidence percentage was calculated as under:

No. of infected plants
$\begin{gathered}\text { Total No. of plants } \\ \text { The resistance against disease was evaluated }\end{gathered}$ 
by Akhtar et al., 2012.

\section{Results and Discussion}

\section{Screening of planting materials}

One hundred genotypes were screened during winter season under field condition and data was recorded on $0-5$ scale at the 30,60 and 90 days. Result showed that the 11, 17, 19, 24 and 29 genotypes were categorized as highly resistant (PDI 0.01 - 10), resistant (PDI 10.01 - 25), moderately resistant (PDI 25.01 - 40), susceptible (PDI 40.01 - 60) and highly susceptible (PDI > 60.01), respectively (Table 1). These results were in agreement with the findings of Gopal and Singh (2003), Govers (2005), Irzhansky and Cohen (2006), Nowicki et al., (2012), Nowicki et al., (2013), Forbes et al., (2014) and Nowakowska et al., (2014).

\section{Screening of parental lines and hybrids}

Forty eight $F_{1} s$ were screened along with their parents during rainy season, 2014 under field condition. Data presented in Table 2 indicated that the parents namely, Solanum peruvianum and Pusa Rohini showed highly resistant and parents viz., IC-395457 and IC-395461 showed resistant disease reaction against late blight of tomato. Other parents were either moderately resistant, susceptible or highly susceptible in respect of disease reaction. Table 2 also showed that out of forty eight $F_{1} s$, eighteen cross combinations namely, Arka Vikas $\times$ IC-395457, LA-3976 × IC-395457, LA-3938 $\times$ IC-395457, LA-3962 $\times$ IC395457, LA-3948 $\times$ IC-395457, Arka Vikas $\times$ S. peruvianum, $\mathrm{H}-86 \times S$. peruvianum, Arka Meghali $\times$ S. peruvianum, LA-3976 $\times$ S. peruvianum, LA-3952 $\times$ S. peruvianum, LA$3930 \times$ S. peruvianum, Arka Vikas $\times$ IC395461, LA-3976 × IC-395461, LA-3962 × IC-395461, LA-3930 $\times$ IC-395461, LA-3948 $\times$ IC-395461, H-86 $\times$ Pusa Rohini and LA$3948 \times$ Pusa Rohini were highly resistant whereas nine cross combinations Pant-T-5 $x$ IC-395457, LA-3967 $\times$ S. peruvianum, LA$3938 \times S$. peruvianum, Pant-T-5 $\times S$. peruvianum, H-86 $\times$ IC-395461, Arka Meghali $\times$ IC-395461, LA-3938 $\times$ Pusa Rohini, LA-3962 × Pusa Rohini and H-88-78$5 \times$ Pusa Rohini were resistant.

Disease rating scale

\begin{tabular}{|l|c|c|l|}
\hline \multicolumn{1}{|c|}{ Symptoms } & Disease rating & Infection \% & \multicolumn{1}{|c|}{ Reaction } \\
\hline No visible symptoms apparent & 0 & 0 & Immune \\
\hline $\begin{array}{l}\text { A few minute lesions to about 10\% of } \\
\text { the total leaf area is blighted and } \\
\text { usually confined to the 2 bottom } \\
\text { leaves. }\end{array}$ & 1 & $0.01-10$ & $\begin{array}{l}\text { Highly } \\
\text { resistant }\end{array}$ \\
\hline $\begin{array}{l}\text { Leaves on about } \mathbf{2 5 \%} \text { of the total } \\
\text { plant area are infected. }\end{array}$ & 2 & $10.01-25$ & Resistant \\
\hline $\begin{array}{l}\text { Leaves on about } \mathbf{5 0 \%} \text { of the total } \\
\text { plant area are infected. }\end{array}$ & 3 & $25.01-40$ & $\begin{array}{l}\text { Moderately } \\
\text { resistant }\end{array}$ \\
\hline $\begin{array}{l}\text { Leaves on about } \mathbf{7 5 \%} \text { of the total } \\
\text { plant area are infected. }\end{array}$ & 4 & $40.01-60$ & \begin{tabular}{l} 
Susceptible \\
\hline $\begin{array}{l}\text { Leaves on whole plant are blighted } \\
\text { and plant is dead. }\end{array}$
\end{tabular} \\
\hline
\end{tabular}


Table.1 Natural screening of one hundred genotypes during winter season, 2012

\begin{tabular}{|c|c|c|c|}
\hline $\begin{array}{l}\text { Disease } \\
\text { scale }\end{array}$ & Reaction & $\begin{array}{l}\text { Total number of } \\
\text { genotypes }\end{array}$ & Name of Genotypes \\
\hline 0.01-10 & HR & 11 & $\begin{array}{l}\text { H-24, IC-395461, F-5020, F-6-1, EC-621667, Azad T-5, S. Peruvianum,B-9-2, EC-538439, EC-538408, H- } \\
\text { 28-78-1 }\end{array}$ \\
\hline $10.01-25$ & $\mathrm{R}$ & 17 & $\begin{array}{l}\text { Bhaillai-2, B-10-2, Pusa Rohini, IC-395457,Arka Saurabh, CLNB, S. Cheesmanii, IIHR-2629,C-19-1, EC- } \\
\text { 366899, Azad T-6, EC-620421, EC-620439, EC-620438, EC-620404, EC-620444, C-6 T }\end{array}$ \\
\hline $25.01-40$ & MR & 19 & $\begin{array}{l}\text { H-86, EC-501580, LA-3948, EC-501582, LA-3952, EC-520046, EC-520075,ArkaVikash, F-7012, LA- } \\
\text { 3930, EC-528374, EC-538380, EC-501577, Pusa Ruby, EC-676781, IIHR-2755, EC-677068, Arka Abha, } \\
\text { IIHR-2754 }\end{array}$ \\
\hline $40.01-60$ & S & 24 & $\begin{array}{l}\text { EC-538411, CLN-1621-L, DNT-1, Azad T-2, SEL-18, EC-620500, EC-620377, EC-570422, Rio Grande, } \\
\text { EC-538455, Cheku Grande, EC-381263, EC-501575,HATH-8, Hisar Arun, Hisar Lalit, GT-2, H-1-1, } \\
\text { Arka Alok, Arka Ahuti, Arka Anannya, Badshah, Tripura Local, Pant T-7 }\end{array}$ \\
\hline$>60.01$ & HS & 29 & $\begin{array}{l}\text { EC-521080, EC-528372, ArkaMeghali,IIHR-2619, C-26-1, EC-620520, C-7-1, EC-620568, C-22-2, C-9-2, } \\
\text { EC-16788, EC-620541, EC-620564, LA-3967, LA-3976, EC-538156, Pant T-5, EC-538405, H-88-78-5, } \\
\text { EC-529080, LA-3962, EC-620419, EC-620478, LA-3938, EC-620505, EC-620442, VRT-2, H-88-78-4, } \\
\text { IIHR-837 }\end{array}$ \\
\hline
\end{tabular}

Table.2 Natural screening of 16 parents and their 48 F $_{1}$ 's during rainy season, 2013

\begin{tabular}{|c|c|c|c|c|c|}
\hline Disease scale & Reaction & $\begin{array}{l}\text { No. of } \\
\text { parents }\end{array}$ & Parents & $\begin{array}{l}\text { No. of } \\
\text { crosses }\end{array}$ & Crosses \\
\hline 0.01-10 & HR & 02 & $\begin{array}{l}\text { Pusa Rohini, Solanum } \\
\text { peruvianum }\end{array}$ & 18 & $\begin{array}{l}\text { Arka Vikas } \times \text { IC-395457, LA-3976 } \times \text { IC-395457, LA-3938 } \times \text { IC-395457, LA-3962 } \times \text { IC- } \\
395457, \text { LA-3948 } \times \text { IC-395457, Arka Vikas } \times \text { S. peruvianum, H-86 } \times \text { S. peruvianum, } \\
\text { Arka Meghali } \times \text { S. peruvianum, LA-3976 } \times \text { S. peruvianum, LA-3952 } \times \text { S. peruvianum, } \\
\text { LA-3930 } \times \text { S. peruvianum, Arka Vikas } \times \text { IC-395461, LA-3976 } \times \text { IC-395461, LA-3962 } \times \\
\text { IC-395461, LA-3930 } \times \text { IC-395461, LA-3948 } \times \text { IC-395461, H-86 } \times \text { Pusa Rohini, LA-3948 } \\
\times \text { Pusa Rohini }\end{array}$ \\
\hline 10.01-25 & $\mathrm{R}$ & 02 & IC-395461, IC-395457 & 09 & $\begin{array}{l}\text { Pant-T-5 × IC-395457, LA-3967 × S. peruvianum, LA-3938 } \times \text { S. peruvianum, Pant-T-5 } \\
\times \text { S. peruvianum, H-86 } \times \text { IC-395461, Arka Meghali } \times \text { IC-395461, LA-3938 } \times \text { Pusa } \\
\text { Rohini, LA-3962 } \times \text { Pusa Rohini, H-88-78-5 } \times \text { Pusa Rohini }\end{array}$ \\
\hline 25.01-40 & MR & 02 & LA-3938,Pant-T-5, & 05 & $\begin{array}{l}\text { H-86 } \times \text { IC-395457, LA-3967 } \times \text { IC-395457, LA-3952 } \times \text { IC-395457, LA-3967 } \times \text { Pusa } \\
\text { Rohini, LA-3930 } \times \text { Pusa Rohini }\end{array}$ \\
\hline 40.01-60 & S & 02 & Arka Vikas, LA 3930, & 03 & H-88-78-5 × IC-395457, Arka Vikas× Pusa Rohini, LA-3976 × Pusa Rohini \\
\hline$>60.01$ & HS & 08 & $\begin{array}{l}\text { H-86, Arka Meghali, LA- } \\
\text { 3976, LA-3952, LA-3948, LA- } \\
\text { 3967, H-88-78-5, LA-3962, }\end{array}$ & 13 & $\begin{array}{l}\text { Arka Meghalix IC-395457, LA-3930 } \times \text { IC-395457, LA-3962 } \times \text { S. peruvianum, H-88-78- } \\
5 \times \text { S. peruvianum, LA-3948 } \times \text { S. peruvianum, LA-3967 } \times \text { IC-395461, LA-3938 } \times \text { IC- } \\
395461, \text { H-88-78-5 } \times \text { IC-395461, LA-3952 } \times \text { IC-395461, Pant-T-5 } \times \text { IC-395461, Arka } \\
\text { Meghalix Pusa Rohini, LA-3952 } \times \text { Pusa Rohini, Pant-T-5 } \times \text { Pusa Rohini }\end{array}$ \\
\hline
\end{tabular}


Table.3 Disease incidence for late blight of tomato in parents

\begin{tabular}{|c|c|c|c|c|c|}
\hline S. No. & Genotypes & $\begin{array}{l}\text { Severity } \\
\text { Grade }\end{array}$ & $\begin{array}{l}\text { Per cent of } \\
\text { disease } \\
\text { infection }\end{array}$ & $\begin{array}{l}\text { Coefficient of } \\
\text { infection }\end{array}$ & Reaction \\
\hline 1 & 2 & 3 & 5 & 6 & 7 \\
\hline 1. & Arka Vikas & 3 & 67.16 & 50.37 & S \\
\hline 2. & H-86 & 4 & 97.75 & 97.75 & HS \\
\hline 3. & Arka Meghali & 4 & 76.63 & 76.63 & HS \\
\hline 4. & LA-3967 & 4 & 85.31 & 85.31 & HS \\
\hline 5. & LA-3976 & 4 & 79.32 & 79.32 & HS \\
\hline 6. & LA-3938 & 3 & 65.65 & 49.24 & MR \\
\hline 7. & LA-3962 & 4 & 77.07 & 77.07 & HS \\
\hline 8. & H-88-78-5 & 4 & 78.33 & 78.33 & HS \\
\hline 9. & LA-3952 & 4 & 76.24 & 76.24 & HS \\
\hline 10. & LA-3930 & 3 & 74.92 & 56.19 & S \\
\hline 11. & LA-3948 & 4 & 94.15 & 94.15 & HS \\
\hline 12. & Pant-T-5 & 3 & 66.51 & 49.88 & MR \\
\hline 13. & IC-395457 & 1 & 13.82 & 3.46 & $\mathrm{R}$ \\
\hline 14. & S. peruvianum & 1 & 1.38 & 0.35 & HR \\
\hline 15. & IC-395461 & 1 & 19.75 & 4.94 & $\mathrm{R}$ \\
\hline 16. & Pusa Rohini & 1 & 2.42 & 0.61 & HR \\
\hline
\end{tabular}

Table.4 Disease incidence for late blight of tomato in crosses

\begin{tabular}{|c|c|c|c|c|c|}
\hline S. No. & Genotypes & $\begin{array}{l}\text { Severity } \\
\text { Grade }\end{array}$ & $\begin{array}{l}\text { Per cent } \\
\text { disease } \\
\text { infection }\end{array}$ & $\begin{array}{l}\text { Coefficient of } \\
\text { infection }\end{array}$ & Reaction \\
\hline & 2 & 3 & 5 & 6 & 7 \\
\hline 1. & Arka Vikas $\times$ IC-395457 & 1 & 22.47 & 5.62 & HR \\
\hline 2. & H-86 × IC-395457 & 2 & 48.89 & 24.45 & MR \\
\hline 3. & Arka Meghali × IC-395457 & 3 & 55.51 & 41.63 & HS \\
\hline 4. & LA-3967 × IC-395457 & 2 & 44.45 & 22.23 & MR \\
\hline 5. & LA-3976 × IC-395457 & 1 & 16.98 & 4.25 & HR \\
\hline 6. & LA-3938 $\times$ IC-395457 & 1 & 12.86 & 3.22 & HR \\
\hline 7. & LA-3962 × IC-395457 & 1 & 30.22 & 7.56 & HR \\
\hline 8. & H-88-78-5 × IC-395457 & 3 & 52.29 & 39.22 & S \\
\hline 9. & LA-3952 × IC-395457 & 2 & 40.06 & 20.03 & MR \\
\hline 10. & LA-3930 × IC-395457 & 3 & 55.88 & 41.91 & HS \\
\hline 11. & LA-3948 × IC-395457 & 1 & 24.39 & 6.10 & HR \\
\hline 12. & Pant-T-5 × IC-395457 & 2 & 33.86 & 16.93 & $\mathrm{R}$ \\
\hline 13. & Arka Vikas $\times S$. peruvianum & 1 & 16.31 & 4.08 & HR \\
\hline 14. & $\mathrm{H}-86 \times S$. peruvianum & 1 & 1.79 & 0.45 & HR \\
\hline
\end{tabular}




\begin{tabular}{|c|c|c|c|c|c|}
\hline 15. & Arka Meghali $\times S$. peruvianum & 1 & 15.13 & 3.78 & HR \\
\hline 16. & LA-3967 $\times$ S. peruvianum & 2 & 27.08 & 13.54 & $\mathrm{R}$ \\
\hline 17. & LA-3976 $\times$ S. peruvianum & 1 & 23.66 & 5.92 & HR \\
\hline 18. & LA-3938 $\times$ S. peruvianum & 2 & 32.18 & 16.09 & $\mathrm{R}$ \\
\hline 19. & LA-3962 $\times$ S. peruvianum & 3 & 64.21 & 48.16 & HS \\
\hline 20. & H-88-78-5 $\times$ S. peruvianum & 3 & 59.40 & 44.55 & HS \\
\hline 21. & LA-3952 $\times$ S. peruvianum & 1 & 5.31 & 1.33 & HR \\
\hline 22. & LA-3930 $\times S$. peruvianum & 1 & 6.15 & 1.54 & HR \\
\hline 23. & LA-3948 $\times S$. peruvianum & 3 & 64.35 & 48.26 & HS \\
\hline 24. & Pant-T- $5 \times S$. peruvianum & 2 & 32.81 & 16.41 & $\mathrm{R}$ \\
\hline 25. & Arka Vikas $\times$ IC-395461 & 1 & 23.82 & 5.96 & HR \\
\hline 26. & H- $86 \times$ IC-395461 & 2 & 25.57 & 12.79 & $\mathrm{R}$ \\
\hline 27. & Arka Meghali × IC-395461 & 2 & 31.12 & 15.56 & $\mathrm{R}$ \\
\hline 28. & LA-3967 × IC-395461 & 3 & 69.12 & 51.84 & HS \\
\hline 29. & LA-3976 × IC-395461 & 1 & 17.43 & 4.36 & HR \\
\hline 30. & LA-3938 × IC-395461 & 3 & 60.26 & 45.20 & HS \\
\hline 31. & LA-3962 × IC-395461 & 1 & 19.61 & 4.90 & HR \\
\hline 32. & H-88-78-5 × IC-395461 & 3 & 56.33 & 42.25 & HS \\
\hline 33. & LA-3952 × IC-395461 & 3 & 55.66 & 41.75 & HS \\
\hline 34. & LA-3930 $\times$ IC-395461 & 1 & 19.13 & 4.78 & HR \\
\hline 35. & LA-3948 ×IC-395461 & 1 & 13.45 & 3.36 & HR \\
\hline 36. & Pant-T-5 × IC-395461 & 3 & 55.60 & 41.70 & HS \\
\hline 37. & Arka Vikas $\times$ Pusa Rohini & 3 & 51.35 & 38.51 & $\mathrm{~S}$ \\
\hline 38. & H-86 × Pusa Rohini & 1 & 13.11 & 3.28 & HR \\
\hline 39. & Arka Meghali $\times$ Pusa Rohini & 3 & 55.73 & 41.80 & HS \\
\hline 40. & LA-3967 ×Pusa Rohini & 2 & 43.02 & 21.51 & MR \\
\hline 41. & LA-3976 $\times$ Pusa Rohini & 3 & 53.31 & 39.98 & $\mathrm{~S}$ \\
\hline 42. & LA-3938 $\times$ Pusa Rohini & 2 & 34.70 & 17.35 & $\mathrm{R}$ \\
\hline 43. & LA-3962 $\times$ Pusa Rohini & 2 & 35.45 & 17.73 & $\mathrm{R}$ \\
\hline 44. & H-88-78-5 ×Pusa Rohini & 2 & 29.10 & 14.55 & $\mathrm{R}$ \\
\hline 45. & LA-3952 $\times$ Pusa Rohini & 3 & 53.78 & 40.34 & HS \\
\hline 46. & LA-3930 $\times$ Pusa Rohini & 2 & 46.69 & 23.35 & MR \\
\hline 47. & LA-3948 $\times$ Pusa Rohini & 1 & 1.28 & 0.32 & HR \\
\hline 48. & Pant-T-5 $\times$ Pusa Rohini & 3 & 57.60 & 43.20 & HS \\
\hline
\end{tabular}

Five cross combinations were moderately resistant viz., H-86 × IC-395457, LA-3967 × IC-395457, LA-3952 × IC-395457, LA-3967 $\times$ Pusa Rohini and LA-3930 $\times$ Pusa Rohini whereas, some susceptible H-88-78-5 $\times$ IC395457, Arka Vikas $\times$ Pusa Rohini, LA-3976 $\times$ Pusa Rohini and highly susceptible Arka Meghali $\times$ IC-395457, LA-3930 × IC-395457, LA-3962 $\times$ S. peruvianum, H-88-78-5 $\times S$. peruvianum, LA-3948 $\times$ S. peruvianum, LA$3967 \times$ IC-395461, LA-3938 $\times$ IC-395461, H88-78-5 × IC-395461, LA-3952 × IC-395461, Pant-T-5 $\times$ IC-395461, Arka Meghali $\times$ Pusa Rohini, LA-3952 $\times$ Pusa Rohini and Pant-T-5 $\times$ Pusa Rohini.

The coefficient of infection of late blight of tomato was recorded in the range of $0.35 \%$ 
(S. peruvianum) to $97.75 \%(\mathrm{H}-86)$ in parental lines (Table 3) and $0.32 \%$ (LA-3948 $\times$ Pusa Rohini) to $51.84 \%($ LA-3967 $\times$ IC-395461) in crosses (Table 4). Among the parents $S$. peruvianum, Pusa Rohini, IC-395457 and IC395461 were highly resistant due to low coefficient of infection. However, among the crosses Arka Vikas $\times$ IC-395457, LA-3976 × IC-395457, LA-3938 × IC-395457, LA-3962 $\times$ IC-395457, LA-3948 × IC-395457, Arka Vikas $\times S$. peruvianum, $\mathrm{H}-86 \times S$. peruvianum, Arka Meghali $\times S$. peruvianum, LA-3976 $\times S$. peruvianum, LA-3952 $\times S$. peruvianum, LA-3930 $\times$ S. peruvianum, Arka Vikas $\times$ IC-395461, LA-3976 × IC-395461, LA-3962 $\times$ IC-395461, LA-3930 $\times$ IC 395461, LA-3948 × IC-395461, H-86 × Pusa Rohini and LA-3948 $\times$ Pusa Rohini were very low coefficient of infection.

\section{References}

Afroz, A., Z. Chaudhry., R. Khan., H. Rashid and S. A. Khan 2009. Effect of $\mathrm{GA}_{3}$ on regeneration response of three tomato cultivars (Lycopersicon esculentum). Pak. J. Bot., 41: 143-151.

Akhtar, K. P., Saleem, M. Y., Asghar, M., Ali, S., Sarwar, N., Elahi, M. T. 2012. Resistance of Solanum species to Phytophthora infestans evaluated in the detached leaf assays and whole plant assays. Pak. J. of Bot. 44(3): 11411146.

Bectell, M. C., C. D. Smart., C. H. Haney and W. E. Fry 2006. Host pathogen interaction between Phytophthora infestans and the solanaceous hosts Calibrachoa $\times$ hybridus, Petunia $\times$ hybrida and Nicotiana benthamiana. Plant Dis. 90:24-32.

Dandurand, L. M., G. R. Kundsen and C. V. Eberlein 2006. Susceptibility of five nightshade (Solanum) species to Phytophthora infestans. Amer. J. Potato Res., 83: 205-210.
Flier, W. G., G. M. B. van der Bosch and L. J Turkensteen 2003. Epidemological importance of Solanum sisymbriifolium, $S$. nigrum and $S$. dulcamara as alternative hosts for Phytophthora infestans. Plant Pathol, 52: 595-603.

Forbes, G., Pérez, W. and Andrade Piedra J. 2014. Field assessment of resistance in potato to Phytophthora infestans. International Potato Center (CIP), Lima (Peru).pp 1-35.

Gopal, J. and Singh, B. 2003. Screening potato for resistance to late blight (Phytophthora infestans) under field conditions. Potato Research 46: 47-56.

Govers, F. 2005. Late blight: The perspective from the pathogen. In: (Eds.): A. J. Havenkort and P. C. Strik, Potato in progress: Science meets practice. The Netherlands: Wageningen Academic Publishers. pp. 245-254.

Griffith, J. M., A. J. Davis and B. R. Grant 1992. Target sites of fungicides to control oomycetes. In Target Sites of Fungicide Action. (Ed.): W. Koller, London CRC Press. pp. 69-100.

Irzhansky, I. and Y. Cohen 2006. Inheritance of resistance against Phytophthora infestans in Lycopersicon pimpinellifolium L3707. Euphy., 149: 309-316.

Lebecka, R. 2008. Host-pathogen interaction between Phytophthora infestans and Solanum nigrum, $S$. villosum and $S$. scabum. Europ J. Plant Pathol., 120: 233-240.

Majid, K., M. Aslam., M. Shahid and A. Saleem 1992. Late blight of tomato caused by Phytophthora infestans (Mont.) de Bary. A new record for Pakistan. Pak. J. Phytopathol. 4:70.

Mohan, S. K., M. K. Thornton., P. Nolte and V. P. Bijm 1996. Late blight of potato and tomato. University of Idaho, College of Agriculture. Cooperative Extension System. Publication. CIS 
1051.

Noureen, F., M. S. Jilani., K. Waseem and M.

Kiran 2010. Performance of tomato hybrids under hydroponic culture. Pak. J. Agri. Sci., 47: 19-25.

Nowakowska, M., Nowicki, M., Kłosinska, U., Maciorowski, R. and Kozik, E. U. 2014. Appraisal of artificial screening techniques of tomato to accurately reflect field performance of the late blight resistance. PLoS ONE 9(10): e109328.

Nowicki, M., Foolad, M. R., Nowakowska, M. and Kozik, E. U. 2012. Potato and tomato late blight caused by Phytophthora infestans: An overview of pathology and resistance breeding. Plant Disease.96: 4-17.

Nowicki, M., Kozik, E. U. and Foolad, M. R. 2013. Late blight of tomato. (In) Translational Genomics for Crop Breeding, pp 241-65.Varshney R K and Tuberosa R (Eds). John Wiley and Sons Ltd.

Payen, 1847. Végétation du Botrytis infestansà l'interieur des fruits du Solanum lycopersicum, erythrocarpum (tomate). Compt. Rend. Acad. Sci. Paris,
25:521-524.

Rubin, E. and Y. Cohen 2004. Oospores associated with tomato seed may lead to seed-borne transmission of Phytophthora infestans. Phytopara., 32: 237-245.

Saleem, M. Y., M. Asghar., M. A. Haq., T. Rafique., A. Kamran and A. A. Khan 2009. Genetic analysis to identify suitable parents for hybrid seed production in tomato (Lycopersicon esculentum Mill.)Pak. J. Bot., 41(3): 1107-1116.

Stevenson, R. W. 1997. Late blight: In: (Eds.): J. B. Jones, J. P. Jones, R. E. Stall and T. A. Zitter. Compendium of tomato diseases. The American Phytopath.Society.3340. Pilot Knob Road, Minnesota 55121-2097, U SA.pp17-18.

Yan, Z., M. S. Reddy., C-M. Ryu, J. A. McInroy., M. Wilson and J. W. Kloepper 2002. Induced systemic protection against tomato late blight elicited by plant growth-promoting rhizobacteria. Phytopathol., 92: 13291333.

\section{How to cite this article:}

Ray, P.K., R.B. Verma, S.S. Solankey and Chaudhary, A. 2018. Assessment of Tomato Advanced Lines to Resistance of Late Blight. Int.J.Curr.Microbiol.App.Sci. 7(01): 2622-2629. doi: https://doi.org/10.20546/ijcmas.2018.701.313 